\title{
Implications of $\theta_{13}$ on Fritzsch-like lepton mass matrices
}

\author{
Priyanka Fakay $^{1}$, Samandeep Sharma ${ }^{1}$, Rohit Verma ${ }^{2}$, \\ Gulsheen Ahuja ${ }^{1 *}$, Manmohan Gupta ${ }^{1}$ \\ ${ }^{1}$ Department of Physics, Centre of Advanced Study, P.U., Chandigarh, India. \\ ${ }^{2}$ Rayat Institute of Engineering and Information Technology, Ropar, India. \\ ( ${ }^{*}$ email: gulsheenahuja@yahoo.co.in)
}

February 8, 2018

\begin{abstract}
Implications of the lepton mixing angles, in particular of $\theta_{13}$, have been investigated for minimal as well as non-minimal Fritzsch-like textures for the case of Majorana neutrinos. Both, in minimal texture (texture 6 zero lepton mass matrices) and non-minimal textures (two cases of texture 5 zero lepton mass matrices), inverted hierarchy and degenerate scenario of neutrino masses have been ruled out. The implications of $\theta_{13}$ have been investigated on the lightest neutrino mass $m_{\nu_{1}}$ as well as the effective Majorana mass $\left\langle m_{e e}\right\rangle$.
\end{abstract}

Keywords: Lepton mass matrices, mixing angle $\theta_{13}$.

PACS numbers: 12.15.Ff, 14.60.Pq

\section{Introduction}

In the last few years, impressive advances have been made in understanding the phenomenology of neutrino oscillations through solar neutrino experiments [1]-[7], atmospheric neutrino experiments [8], reactor based experiments [9],[10] and accelerator based experiments [11]-[13]. The recent measurements of angle $\theta_{13}$ [14]-[18] have undoubtedly improved 
our understanding of the neutrino mixing matrix. Interestingly a not so small value of $\theta_{13}$ which is close to CHOOZ [19] bound and to the Cabibbo angle, coupled with the fact that $\theta_{23}$ may not be maximal [20], 21], strongly indicates that there may not be any symmetry in the leptonic sector [22]. These observations have also deepened the mystery of flavor mixings as the pattern of quark and lepton mixing angles now looks to be significantly different. Since the mixing angles are related to the corresponding mass matrices, therefore, formulating viable fermion mass matrices becomes all the more complicated especially when quarks and leptons have to be described in a unified framework.

In the absence of any compelling theory of flavor dynamics to explain the fermion masses and mixings, the present day approaches can be broadly categorized into 'topdown' and 'bottom-up'. The top-down approach consists of formulating the fermion mass matrices at Grand Unified Theories (GUT) scale based on certain fundamental theoretical principles. However, in the absence of any such viable approach, it is desirable to follow the bottom-up approach. This approach consists of finding the phenomenological fermion mass matrices which are in tune with the low energy data, thereby providing hints for the formulation of mass matrices at the GUT scale. As an example of this approach, texture specific mass matrices, introduced implicitly by Weinberg [23] and explicitly by Fritzsch [24], have played an important role in understanding the pattern of quark and lepton mixing phenomena [25].

Recently, in the light of observation of neutrino mixing angle $\theta_{13}$, Fukugita et al. [26] (henceforth referred to as FSTY) have updated their previous analysis [27] of texture 6 zero Fritzsch-like lepton mass matrices for normal hierarchy of neutrino masses and have arrived at some interesting conclusions. In particular, considering Majorana neutrinos with normal hierarchy of neutrino masses as well as the recent measurements of angle $\theta_{13}$ the authors find the mass of the lightest neutrino to be $0.7-2.1 \mathrm{meV}$. Further, the effective Majorana mass $\left\langle m_{e e}\right\rangle$, appearing in the double beta decay, comes out to be 3.7$5.6 \mathrm{meV}$. Also, it has been shown that the sum of the masses of the three neutrinos is $61 \pm 2 \mathrm{meV}$. Furthermore, FSTY without getting into details, have also concluded that inverted hierarchy and degenerate scenario of the neutrino masses are ruled out.

Recently, in the case of quarks it has been shown [28] that not only Fritzsch-like but also non Fritzsch-like texture 6 zero mass matrices have been ruled out. Therefore, in case one has to formulate quark and lepton mass matrices on the same footing, as advocated by Smirnov [29], perhaps there is a need to go beyond texture 6 zero mass matrices even for the leptons. Further, recent measurements of angle $\theta_{13}$ as well as continuous refinements of the other two mixing angles and corresponding mass square differences also provide the necessary motivation to go beyond the minimal texture considered by FSTY .

The purpose of the present communication, on the one hand, is to rule out inverted hierarchy and degenerate scenario of neutrino masses corresponding to texture 6 zero 
lepton mass matrices. On the other hand, keeping in mind that the texture 5 zero mass matrices are not ruled out completely in the quark sector, we have made an attempt to extend the analysis of FSTY to texture 5 zero Fritzsch-like lepton mass matrices with an emphasis on the implications of angle $\theta_{13}$. In particular, we have carried out a detailed analysis pertaining to hierarchies of neutrino masses as well as the implications of $\theta_{13}$ and the other two mixing angles on the lightest neutrino mass for the two possible cases of texture 5 zero lepton mass matrices.

The detailed plan of the paper is as follows. In Section (2), we detail the essentials of the formalism regarding the texture specific mass matrices. Inputs used in the present analysis have been given in Section (3) and the results and discussion have been presented in Section (44). Finally, Section (5) presents a few comments and summarizes our conclusions.

\section{Texture specific mass matrices and the PMNS ma- trix}

To define the various texture specific cases considered here, we begin with the modified Fritzsch-like mass matrices, e.g.,

$$
M_{l}=\left(\begin{array}{ccc}
0 & A_{l} & 0 \\
A_{l}^{*} & D_{l} & B_{l} \\
0 & B_{l}^{*} & C_{l}
\end{array}\right), \quad M_{\nu D}=\left(\begin{array}{ccc}
0 & A_{\nu} & 0 \\
A_{\nu}^{*} & D_{\nu} & B_{\nu} \\
0 & B_{\nu}^{*} & C_{\nu}
\end{array}\right)
$$

$M_{l}$ and $M_{\nu D}$ respectively corresponding to Dirac-like charged lepton and neutrino mass matrices. Both the matrices are texture 2 zero type with $A_{l(\nu)}=\left|A_{l(\nu)}\right| e^{i \alpha_{l(\nu)}}$ and $B_{l(\nu)}=$ $\left|B_{l(\nu)}\right| e^{i \beta_{l(\nu)}}$, in case these are symmetric then $A_{l(\nu)}^{*}$ and $B_{l(\nu)}^{*}$ should be replaced by $A_{l(\nu)}$ and $B_{l(\nu)}$, as well as $C_{l(\nu)}$ and $D_{l(\nu)}$ should respectively be defined as $C_{l(\nu)}=\left|C_{l(\nu)}\right| e^{i \gamma_{l(\nu)}}$ and $D_{l(\nu)}=\left|D_{l(\nu)}\right| e^{i \omega_{l(\nu)}}$.

The texture 6 zero matrices can be obtained from the above mentioned matrices by taking both $D_{l}$ and $D_{\nu}$ to be zero, which reduces the matrices $M_{l}$ and $M_{\nu D}$ each to texture 3 zero type. Texture 5 zero matrices can be obtained by taking either $D_{l}=0$ and $D_{\nu} \neq 0$ or $D_{\nu}=0$ and $D_{l} \neq 0$, thereby, giving rise to two possible cases of texture 5 zero matrices, referred to as texture 5 zero $D_{l}=0$ case pertaining to $M_{l}$ texture 3 zero type and $M_{\nu D}$ texture 2 zero type and texture 5 zero $D_{\nu}=0$ case pertaining to $M_{l}$ texture 2 zero type and $M_{\nu D}$ texture 3 zero type.

To facilitate the understanding of inverted hierarchy case and its relationship to the normal hierarchy case, we detail the essentials of formalism connecting the mass matrix to 
the neutrino mixing matrix. For detailed diagonalization procedure we refer the readers to our earlier work [30], however, to make the manuscript readable we outline the essentials. Texture specific hermitian mass matrix $M_{k}$, where $k=l, \nu D$, can be expressed as

$$
M_{k}=P_{k}^{\dagger} M_{k}^{r} P_{k}
$$

where $M_{k}^{r}$ is a real symmetric matrix with real eigenvalues and $P_{k}^{\dagger}$ and $P_{k}$ are diagonal phase matrices. The matrix $M_{k}^{r}$ is diagonalized by the orthogonal transformation $O_{k}$ yielding

$$
M_{k}=Q_{k} O_{k} \xi_{k} M_{k}^{\operatorname{diag}} O_{k}^{T} P_{k}
$$

wherein, to facilitate the construction of diagonalizing transformations for different hierarchies, we have introduced $\xi_{k}$ defined as $\operatorname{diag}\left(1, e^{i \pi}, 1\right)$ for the case of normal hierarchy and as $\operatorname{diag}\left(1, e^{i \pi}, e^{i \pi}\right)$ for the case of inverted hierarchy.

The charged leptons case is fairly straight forward, whereas in the case of neutrinos, the diagonalizing transformation is hierarchy specific as well as requires some fine tuning of the phases of the right handed neutrino mass matrix $M_{R}$. Noting $k=\nu D$ and using see-saw mechanism

$$
M_{\nu}=-M_{\nu D}^{T}\left(M_{R}\right)^{-1} M_{\nu D}
$$

we can write

$$
M_{\nu}=-P_{\nu D} O_{\nu D} M_{\nu D}^{\text {diag }} \xi_{\nu D} O_{\nu D}^{T} Q_{\nu D}^{T}\left(M_{R}\right)^{-1} Q_{\nu D} O_{\nu D} \xi_{\nu D} M_{\nu D}^{\text {diag }} O_{\nu D}^{T} P_{\nu D}
$$

Assuming fine tuning, the phase matrices $Q_{\nu D}^{T}$ and $Q_{\nu D}$ along with $-M_{R}$ can be taken as $m_{R} \operatorname{diag}(1,1,1)$ as well as using the unitarity of $\xi_{\nu D}$ and orthogonality of $O_{\nu D}$, the above equation can be expressed as

$$
M_{\nu}=P_{\nu D} O_{\nu D} \frac{\left(M_{\nu D}^{\text {diag }}\right)^{2}}{\left(m_{R}\right)} O_{\nu D}^{T} P_{\nu D}
$$

The lepton mixing matrix, in terms of the matrices used for diagonalizing the mass matrices $M_{l}$ and $M_{\nu}$, can be easily obtained and is expressed as

$$
U=\left(Q_{l} O_{l} \xi_{l}\right)^{\dagger}\left(P_{\nu D} O_{\nu D}\right)
$$

Eliminating the phase matrix $\xi_{l}$ by redefinition of the charged lepton phases, the above equation becomes

$$
U=O_{l}^{\dagger} Q_{l} P_{\nu D} O_{\nu D}
$$


where $Q_{l} P_{\nu D}$, without loss of generality, can be taken as $\left(e^{i \phi_{1}}, 1, e^{i \phi_{2}}\right), \phi_{1}$ and $\phi_{2}$ being related to the phases of mass matrices and can be treated as free parameters.

To understand the relationship between diagonalizing transformations for different hierarchies of neutrino masses and for the charged lepton case, we present the diagonalizing transformation $O_{k}$, whose first element can be written as

$$
O_{k}(11)=\sqrt{\frac{m_{2} m_{3}\left(m_{3}+m_{2}-D_{l(\nu)}\right)}{\left(m_{1}+m_{2}+m_{3}-D_{l(\nu)}\right)\left(m_{1}-m_{3}\right)\left(m_{1}-m_{2}\right)}},
$$

where $m_{1}, m_{2}, m_{3}$ are eigenvalues of $M_{k}$. In the case of charged leptons, because of the hierarchy $m_{e} \ll m_{\mu} \ll m_{\tau}$, the mass eigenstates can be approximated respectively to the flavor eigenstates, as has been considered by several authors [31, 32]. In this approximation, $m_{l 1} \simeq m_{e}, m_{l 2} \simeq m_{\mu}$ and $m_{l 3} \simeq m_{\tau}$, one can obtain the first element of the matrix $O_{l}$ from the above element given in equation (9), by replacing $m_{1}, m_{2}, m_{3}$ by $m_{e},-m_{\mu}, m_{\tau}$, e.g.,

$$
O_{l}(11)=\sqrt{\frac{m_{\mu} m_{\tau}\left(m_{\tau}-m_{\mu}-D_{l}\right)}{\left(m_{e}-m_{\mu}+m_{\tau}-D_{l}\right)\left(m_{\tau}-m_{e}\right)\left(m_{e}+m_{\mu}\right)}} .
$$

Equation (9) can also be used to obtain the first element of diagonalizing transformation for Majorana neutrinos, assuming normal hierarchy, defined as $m_{\nu_{1}}<m_{\nu_{2}} \ll m_{\nu_{3}}$, and also valid for the degenerate case defined as $m_{\nu_{1}} \lesssim m_{\nu_{2}} \sim m_{\nu_{3}}$, by replacing $m_{1}, m_{2}$, $m_{3}$ by $\sqrt{m_{\nu 1} m_{R}},-\sqrt{m_{\nu 2} m_{R}}, \sqrt{m_{\nu 3} m_{R}}$, e.g.,

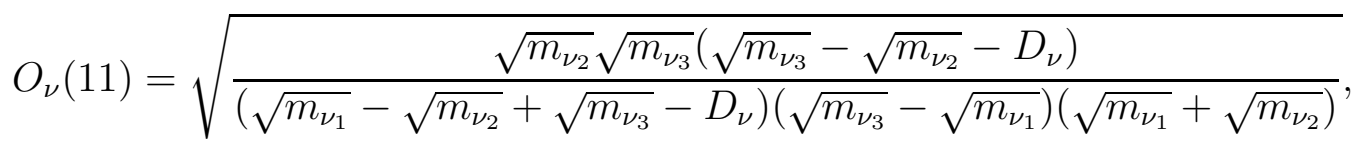

where $m_{\nu_{1}}, m_{\nu_{2}}$ and $m_{\nu_{3}}$ are neutrino masses. The parameter $D_{\nu}$ is to be divided by $\sqrt{m_{R}}$, however as $D_{\nu}$ is arbitrary therefore we retain it as it is.

In the same manner, one can obtain the elements of diagonalizing transformation for the inverted hierarchy case, defined as $m_{\nu_{3}} \ll m_{\nu_{1}}<m_{\nu_{2}}$, by replacing $m_{1}, m_{2}, m_{3}$ in equation (9) with $\sqrt{m_{\nu_{1}} m_{R}},-\sqrt{m_{\nu_{2}} m_{R}},-\sqrt{m_{\nu_{3}} m_{R}}$, e.g.,

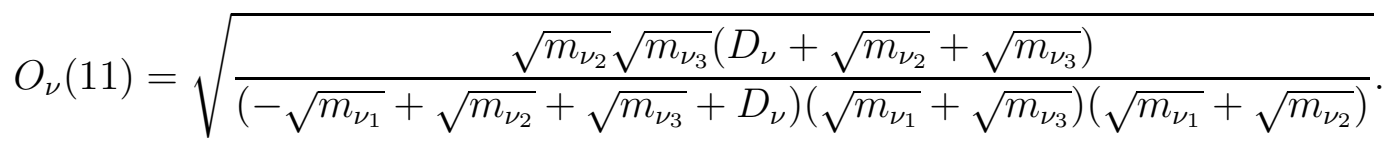

The other elements of diagonalizing transformations in the case of neutrinos as well as 
charged leptons can similarly be found.

\section{Inputs used in present calculations}

Before getting into the details of the analysis, we would like to mention some of the essentials pertaining to various inputs. The present work uses results from the latest global three neutrino oscillation analysis carried out by Fogli et al. [33]. At $1 \sigma$ C.L. the allowed ranges of the various input parameters are

$$
\begin{gathered}
\Delta m_{21}^{2}=(7.32-7.80) \times 10^{-5} \mathrm{eV}^{2}, \quad \Delta m_{23}^{2}=(2.33-2.49) \times 10^{-3} \mathrm{eV}^{2}, \\
s_{12}^{2}=(0.29-0.33), \quad s_{23}^{2}=(0.37-0.41), \quad s_{13}^{2}=(0.021-0.026),
\end{gathered}
$$

where $\Delta m_{i j}^{2}$ 's correspond to the solar and atmospheric neutrino mass square differences and $s_{i j}$ corresponds to the sine of the mixing angle $i j$ where $i, j=1,2,3$. At $3 \sigma$ C.L. the allowed ranges are given as

$$
\begin{aligned}
\Delta m_{21}^{2} & =(6.99-8.18) \times 10^{-5} \mathrm{eV}^{2}, \quad \Delta m_{23}^{2}=(2.19-2.62) \times 10^{-3} \mathrm{eV}^{2}, \\
s_{12}^{2} & =(0.26-0.36), \quad s_{23}^{2}=(0.33-0.64), \quad s_{13}^{2}=(0.017-0.031) .
\end{aligned}
$$

For the purpose of calculations, we have taken the lightest neutrino mass, the phases $\phi_{1}$, $\phi_{2}$ and $D_{l, \nu}$ as free parameters, the other two masses are constrained by $\Delta m_{12}^{2}=m_{\nu_{2}}^{2}-m_{\nu_{1}}^{2}$ and $\Delta m_{23}^{2}=m_{\nu_{3}}^{2}-m_{\nu_{2}}^{2}$ in the normal hierarchy case and by $\Delta m_{23}^{2}=m_{\nu_{2}}^{2}-m_{\nu_{3}}^{2}$ in the inverted hierarchy case. It may be noted that lightest neutrino mass corresponds to $m_{\nu_{1}}$ for the normal hierarchy case and to $m_{\nu_{3}}$ for the inverted hierarchy case. For all the three hierarchies of neutrino masses, the explored range of the lightest neutrino mass is taken to be $10^{-8} \mathrm{eV}-10^{-1} \mathrm{eV}$, however our conclusions remain unaffected even if the range is extended further. The mixing matrix $\mathrm{U}$, given in equation (7) can be constructed in terms of the elements of the transformations $O_{k}$ and $O_{\nu}$ as well as in terms of the phases $\phi_{1}$ and $\phi_{2}$. In the absence of $\mathrm{CP}$ violation in the leptonic sector, phases have been given full variation from 0 to $2 \pi$ to obtain the viable ranges of the mixing angles. In carrying this fit we have constrained $D_{l, \nu}$, the free parameters, such that diagonalizing transformations $O_{l}$ and $O_{\nu}$ always remain real. This implies, for leptons $-\left(m_{l_{2}}-m_{l_{1}}\right)<D_{l}<\left(m_{l_{3}}+m_{l_{2}}\right)$, for Majorana neutrinos $-\left(\sqrt{m_{\nu_{2}}}-\sqrt{m_{\nu_{1}}}\right)<D_{\nu}<\left(\sqrt{m_{\nu_{3}}}-\sqrt{m_{\nu_{2}}}\right)$ for normal hierarchy and $-\left(\sqrt{m_{\nu_{2}}}-\sqrt{m_{\nu_{1}}}\right)<D_{\nu}<\left(\sqrt{m_{\nu_{1}}}-\sqrt{m_{\nu_{3}}}\right)$ for inverted hierarchy. 


\section{Results and discussion}

\subsection{Inverted hierarchy of neutrino masses}

(a)

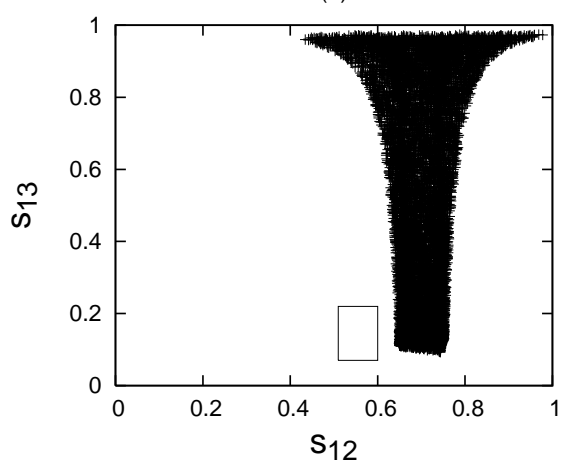

(c)

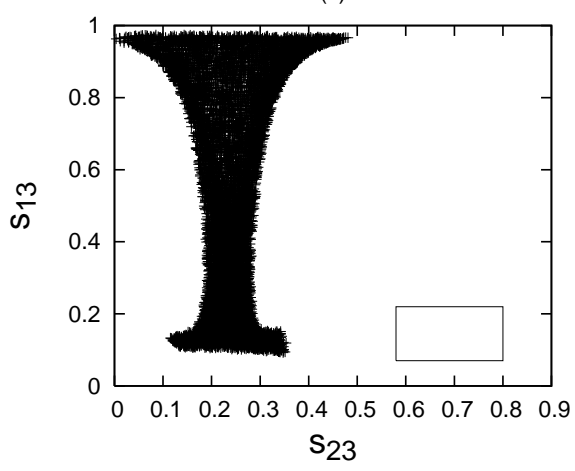

(b)

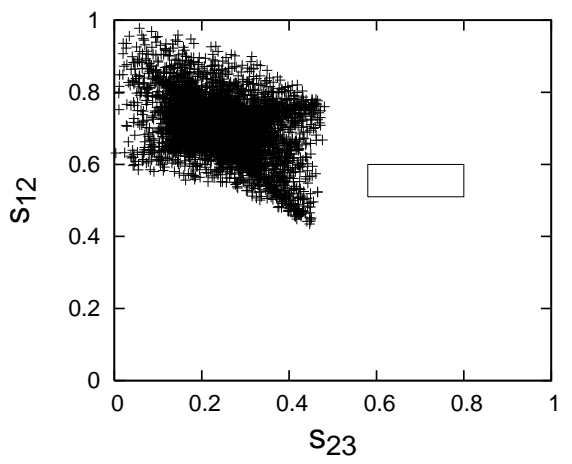

Figure 1: Plots showing the parameter space corresponding to sines of any of the two mixing angles for texture 6 zero lepton mass matrices. The blank rectangular regions indicate the experimentally allowed $3 \sigma$ C.L. region of the plotted angles.

To begin with, we discuss the case of inverted hierarchy of neutrino masses for texture 6 zero as well as texture 5 zero lepton mass matrices. As a first step, in the case of texture 6 zero mass matrices, we have made an attempt to explicitly show the ruling out of inverted hierarchy of neutrino masses, thereby reinforcing the conclusions of FSTY [26]. To this end in Figures (1 $a),(\mathbb{1} b)$ and $(\mathbb{1} c)$, we have plotted the parameter space corresponding to sines of any of the two mixing angles by constraining the third angle by its experimental bound given in equation (16) and giving full allowed variation to other parameters. Also included in the figures are blank rectangular regions indicating the experimentally allowed $3 \sigma$ C.L. region of the plotted angles. Interestingly, a general look at these figures reveals 


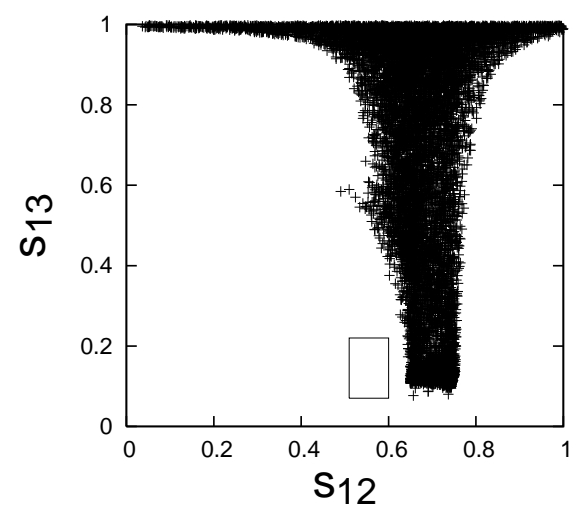

Figure 2: Plot showing the parameter space corresponding to $s_{12}$ versus $s_{13}$ for the $D_{l}=0$ case of texture 5 zero lepton mass matrices. The blank rectangular region indicates the experimentally allowed $3 \sigma$ C.L. region of the plotted angles.

that inverted hierarchy is clearly ruled out. In particular, from Figure (17) showing the plot of $s_{12}$ versus $s_{13}$, one can immediately conclude that the plotted parameter space of these two angles has no overlap with their experimentally allowed $3 \sigma$ C.L. region. This clearly indicates that at $3 \sigma$ C.L. inverted hierarchy of neutrino masses is not viable. These conclusions are further reinforced from Figures (10) and (1) $\mathrm{c}$ ) wherein we have plotted $s_{23}$ versus $s_{12}$ and $s_{23}$ versus $s_{13}$ respectively. Both the figures also indicate that the plotted parameter space does not include simultaneously the experimental bounds of the sines of the plotted angles. Therefore, one can conclude that inverted hierarchy is ruled out for texture 6 zero neutrino mass matrices.

Coming to the two cases of Fritzsch-like texture 5 zero mass matrices, we first discuss the case when $D_{l}=0$ and $D_{\nu} \neq 0$. Primarily to facilitate comparison with texture 6 zero case, in Figure (2) we have plotted $s_{12}$ against $s_{13}$ for inverted hierarchy for a particular value of $D_{\nu}=\sqrt{m_{\nu_{3}}}$. The figure clearly reveals that inverted hierarchy is ruled out as again the plotted parameter space has no overlap with the experimental $3 \sigma$ C.L. ranges of $s_{12}$ and $s_{13}$. For the second case of texture 5 zero mass matrices pertaining to $D_{\nu}=0$ and $D_{l} \neq 0$ case, one can again plot figures similar to the ones shown in Figures (11) and (21). Interestingly, these graphs are essentially similar as shown in Figure (1), therefore these are not presented here. This can be understood by the fact that there is a very strong hierarchy in the case of charged leptons which reduces the texture 5 zero $D_{\nu}=0$ case essentially to texture 6 zero case only. By similar arguments, this case can also be therefore ruled out for inverted hierarchy of neutrino masses. 


\subsection{Degenerate scenario of neutrino masses}

(a)

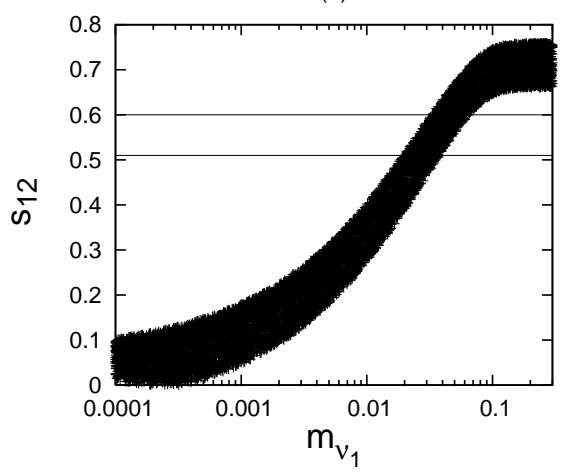

(b)

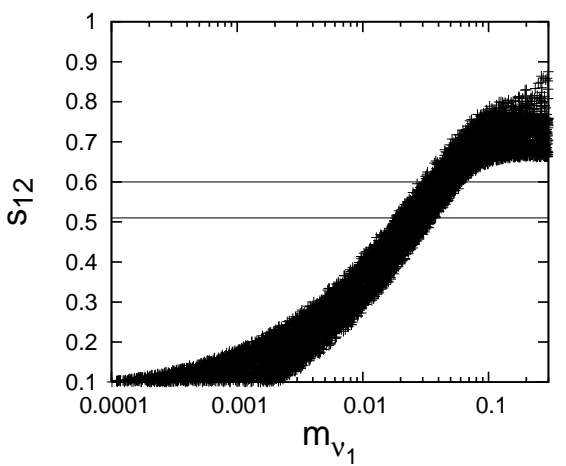

Figure 3: Plot showing the variation of $s_{12}$ with lightest neutrino mass $m_{\nu_{1}}$ for (a) texture 6 zero lepton mass matrices and (b) $D_{l}=0$ case of texture 5 zero lepton mass matrices. The parallel lines indicate the $3 \sigma$ C.L. limits of $s_{12}$.

The cases of neutrino masses being degenerate, characterized by either $m_{\nu_{1}} \lesssim m_{\nu_{2}} \sim$ $m_{\nu_{3}} \lesssim 0.1 \mathrm{eV}$ or $m_{\nu_{3}} \sim m_{\nu_{1}} \lesssim m_{\nu_{2}} \lesssim 0.1 \mathrm{eV}$ corresponding to normal and inverted hierarchy respectively, can again be shown to be ruled out. For texture 6 zero and 5 zero mass matrices considering degenerate scenario corresponding to inverted hierarchy of neutrino masses, Figures (11) and (2) can again be used to rule it out at $3 \sigma$ C.L.. While plotting these figures the range of the lightest neutrino mass is taken to be $10^{-8} \mathrm{eV}-10^{-1} \mathrm{eV}$, which includes the neutrino masses corresponding to degenerate scenario, therefore by discussion similar to the one given for ruling out inverted hierarchy and degenerate scenario of neutrino masses is ruled out as well.

Coming to the degenerate scenario corresponding to normal hierarchy of neutrino masses, one can show that this is ruled out as well. To this end, for texture 6 zero mass matrices in Figure (3 $\mathrm{a}$ ) we have plotted the mixing angle $s_{12}$ against the lightest neutrino mass $m_{\nu_{1}}$. From the figure one can find that the values of $s_{12}$ corresponding to $m_{\nu_{1}} \lesssim 0.1 \mathrm{eV}$ lie outside the experimentally allowed range, thereby ruling out degenerate scenario. For the texture 5 zero mass matrices, pertaining to $D_{l}=0$ case, the degenerate scenario corresponding to normal hierarchy of neutrino masses can again be shown to be ruled out. Similar to the case of texture 6 zero mass matrices Figure ( $3 \mathrm{~b}$ ) again shows that the values of $s_{12}$ corresponding to $m_{\nu_{1}} \lesssim 0.1 \mathrm{eV}$ lie outside the experimentally allowed range. For the $D_{\nu}=0$ case of texture 5 zero mass matrices again one gets essentially the same figures as for the case of texture 6 zero mass matrices therefore degenerate scenario for this case can be ruled out by similar arguments. 


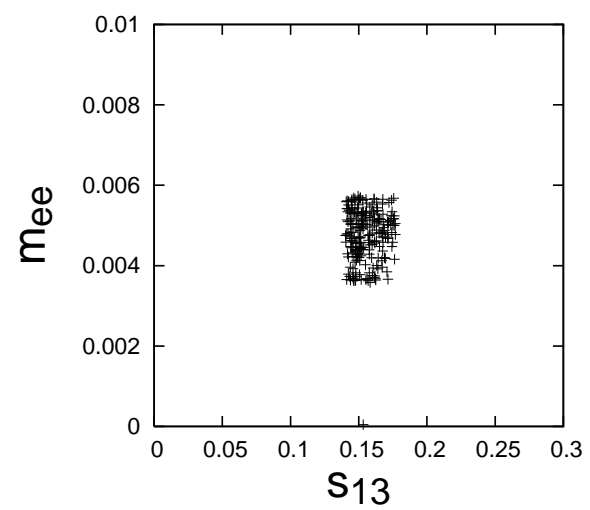

Figure 4: Plot showing variation of effective Majorana mass measured in neutrinoless double beta decay $(\beta \beta)_{0 \nu}$ (in eV) with the mixing angle $s_{13}$ for texture 6 zero lepton mass matrices.

\subsection{Normal hierarchy of neutrino masses}

\subsubsection{Texture 6 zero lepton mass matrices}

To begin with, we have made an attempt to reproduce the results of FSTY [26] for texture 6 zero lepton mass matrices. Using the latest inputs we are largely able to reproduce their results. For the sake of comparison, we have presented our results pertaining to effective Majorana mass measured in neutrinoless double beta decay $(\beta \beta)_{0 \nu}$, given as

$$
\left\langle m_{e e}\right\rangle=m_{\nu_{1}} U_{e 1}^{2}+m_{\nu_{2}} U_{e 2}^{2}+m_{\nu_{3}} U_{e 3}^{2} .
$$

In particular, in Figure (41) we have plotted the variation of $\left\langle m_{e e}\right\rangle$ with respect to the mixing angle $s_{13}$. From the figure it is clear that there is a good overlap between the present calculations and the results of FSTY.

As an extension of the analysis of FSTY for the texture 6 zero lepton mass matrices for normal hierarchy of neutrino masses, in Figures (3a) and 5 (a, b) we have respectively plotted the lightest neutrino mass $m_{\nu_{1}}$ against the mixing angles $s_{12}, s_{13}$ and $s_{23}$. While plotting any of these graphs, the other input parameters have been given variations at $3 \sigma$ C.L.. It may be noted that the plot between $s_{23}$ and $m_{\nu_{1}}$, Figure (5) b) expectedly does not provide any constrains on the range of $m_{\nu_{1}}$ considered here. Similarly, considering the $3 \sigma$ C.L. range of $s_{13}$ we do not get any reasonable constrains on $m_{\nu_{1}}$, however, interestingly because of the almost precise value of $s_{12}$ even at $3 \sigma$ C.L. we get a narrow range of the 
(a)

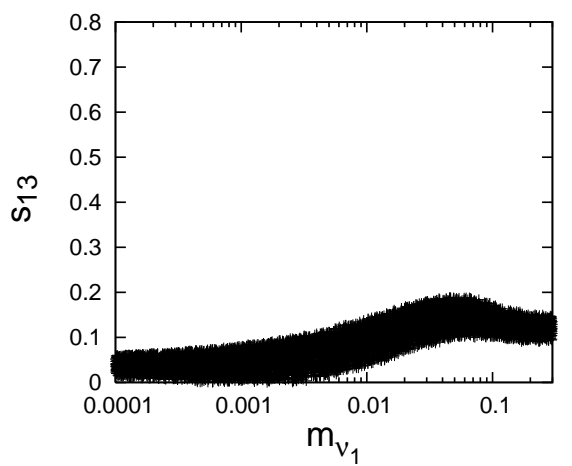

(b)

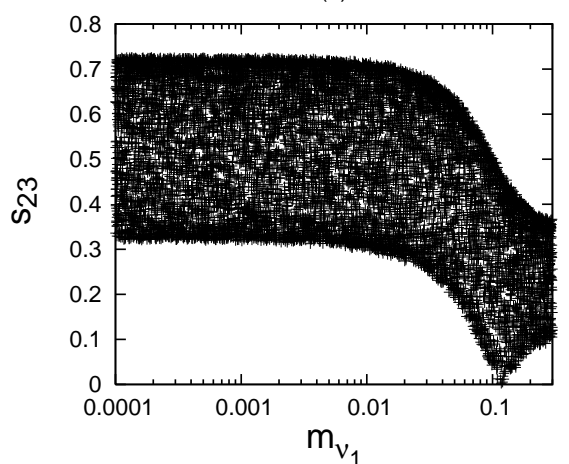

Figure 5: Plots showing variation of the mixing angles $s_{13}$ and $s_{23}$ with the lightest neutrino mass $m_{\nu_{1}}$ for texture 6 zero lepton mass matrices.

lightest neutrino mass $m_{\nu_{1}}=0.03-0.07 \mathrm{eV}$. From Figures (13a) and ( $5 \mathrm{a}$ ) one finds that for $1 \sigma$ C.L. experimental ranges of $s_{12}$ and $s_{13}$ we get the range of $m_{\nu_{1}}$ to be $0.03-0.06 \mathrm{eV}$ and 0.007-0.3 eV respectively. It may be noted that any measurement regarding the lightest neutrino mass $m_{\nu_{1}}$ will have immediate implications for texture 6 zero mass matrices considered here.

\subsubsection{Texture 5 zero mass matrices}

In the case of texture 5 zero lepton mass matrices, we have carried out calculations for both the cases when $D_{l}=0$ with $D_{\nu} \neq 0$ and $D_{\nu}=0$ with $D_{l} \neq 0$. Since the texture 6 zero mass matrices are able to accommodate the lepton mixing data quite well, it is therefore expected that both the cases of texture 5 zero mass matrices would also be viable. Similar to the case of texture 6 zero mass matrices, we would like to study the implications of the mixing angles on the lightest neutrino mass $m_{\nu_{1}}$, in particular that of $\theta_{13}$.

We first discuss the case when $D_{l}=0$ and $D_{\nu} \neq 0$. For normal hierarchy of neutrino masses, the additional parameter $D_{\nu}$ can be constrained such that the diagonalizing transformations $O_{l}$ and $O_{\nu}$ always remain real. Primarily to facilitate comparison with texture 6 zero case, in Figures (3b) and (6) we have plotted the mixing angles against the lightest neutrino mass for normal hierarchy of neutrino masses. Interestingly, we find that this case shows a big change in the behaviour of the mixing angles $s_{13}$ and $s_{23}$ versus the lightest neutrino mass as compared to the texture 6 zero graphs shown in Figure (5). It may be noted that similar to the case of texture 6 zero mass matrices, $s_{13}$ and $s_{23}$ are not able to constrain the lightest neutrino mass $m_{\nu_{1}}$ at $3 \sigma$ C.L.. However at $1 \sigma$ C.L., 
(a)

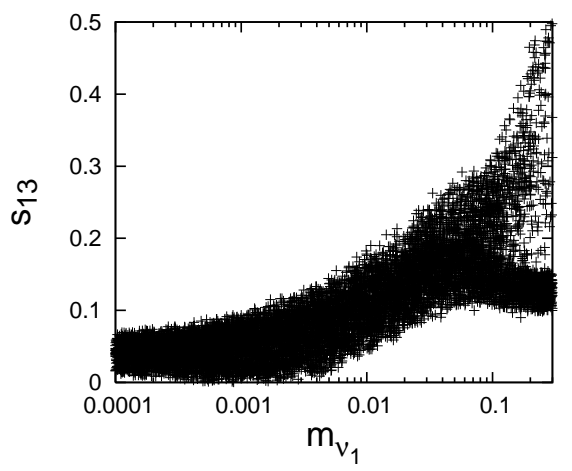

(b)

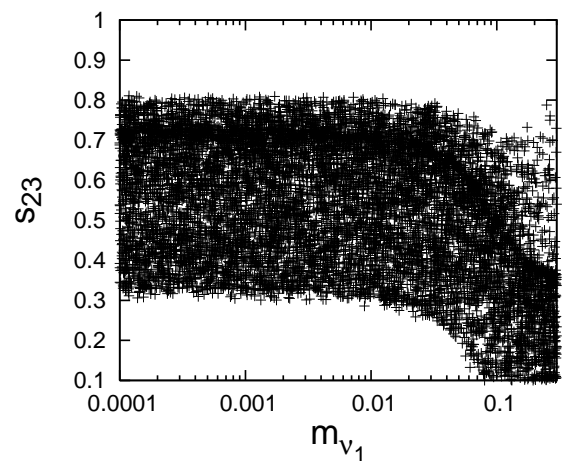

Figure 6: Plots showing variation of mixing angles $s_{13}$ and $s_{23}$ with the lightest neutrino mass $m_{\nu_{1}}$ for texture 5 zero lepton mass matrices when $D_{l}=0$ and $D_{\nu} \neq 0$.

$s_{13}$ constrains the range of $m_{\nu_{1}}$ to be $0.007-0.03 \mathrm{eV}$. Similar to the case of texture 6 zero mass matrices, the present refined value of $s_{12}$, even at $3 \sigma$ C.L., provides the range of the lightest neutrino mass $m_{\nu_{1}}$ as $0.03-0.08 \mathrm{eV}$, which is somewhat expanded in the present case as compared to texture 6 zero mass matrices. This can be clearly understood by noting the fact that $D_{l}=0$ and $D_{\nu} \neq 0$ case introduces additional parameters. It may be added that on comparison with our earlier analysis [34] which was carried out when only an upper bound on $s_{13}$ was known, we find that the present experimental range of $s_{13}$ has sharpened the range of the lightest neutrino mass $m_{\nu_{1}}$.

As a next step, similar to the case of texture 6 zero mass matrices, in Figure (17) we have shown the variation of the mixing angle $s_{13}$ against the effective Majorana mass $\left\langle m_{e e}\right\rangle$. One finds that the $1 \sigma$ C.L. range of the mixing angle $\theta_{13}$ constrains the value of $\left\langle m_{e e}\right\rangle$ to be 2.3-8.7 meV, this being broadly in agreement with [35]. Due to the additional parameter $D_{\nu}$ the range obtained here also shows an expansion in comparison to the one obtained in the case of texture 6 zero mass matrices. It may be of interest to mention that the Jarlskog's rephasing invariant parameter in the leptonic sector $J_{l}$ [36] spans the range $-4.23 \times 10^{-2}-4.36 \times 10^{-2}$.

Coming to the second case of texture 5 zero mass matrices where $D_{\nu}=0$ and $D_{l} \neq 0$, the plots of the mixing angles against the lightest neutrino mass are shown in Figure (8). Interestingly these graphs are very similar to those in Figure (5) corresponding to the texture 6 zero case. This can again be understood by noting the fact that there is very strong hierarchy in the case of charged leptons which reduces the texture 5 zero $D_{\nu}=0$ case essentially to the texture 6 zero case. Also we would like to mention that out of the two free parameters $D_{l}$ and $D_{\nu}$, the parameter $D_{\nu}$ plays a more important role than $D_{l}$. This becomes more clear if we compare the graphs of the texture 6 zero matrices with 


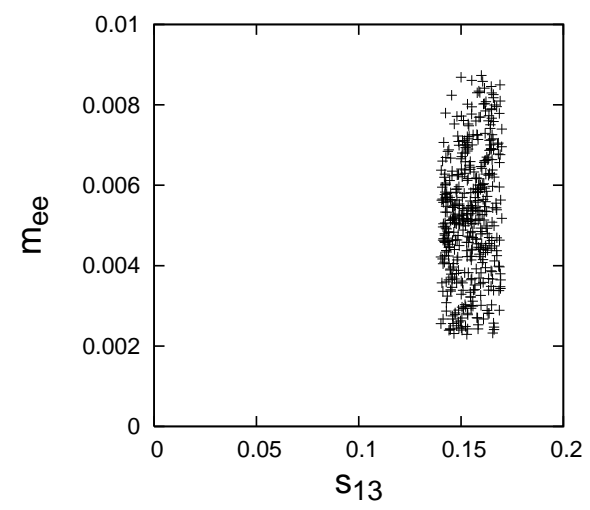

Figure 7: Plot showing variation of effective Majorana mass measured in neutrinoless double beta decay $(\beta \beta)_{0 \nu}$ (in eV) with the mixing angle $s_{13}$ for texture 5 zero lepton mass matrices.

those of the two cases of the texture 5 zero matrices. The texture 6 zero plots and those of the texture 5 zero $D_{\nu}=0$ case are essentially similar whereas those of the texture 5 zero $D_{l}=0$ case, wherein $D_{\nu}$ is non zero, are different from the graphs of the texture 6 zero matrices.

In this case also we present the viable range of Jarlskog's rephasing invariant parameter in the leptonic sector $J_{l}$ which is predicted to be $-4.11 \times 10^{-2}-4.26 \times 10^{2}$. The $\left\langle m_{e e}\right\rangle$ range comes out to be $2.4-6.02 \mathrm{meV}$ which includes the range obtained in texture 6 zero case given as $3.7-5.6 \mathrm{meV}$. In the absence of any definite information about $J_{l}$ and $\left\langle m_{e e}\right\rangle$ we find that the ranges corresponding to different cases are in broad agreement with the similar calculations done by several other authors [35].

\section{Comments, summary and conclusions}

Before summarizing the present work, a few comments are in order. It may be mentioned that in the case when charged leptons are in the flavor basis, the mixing matrix becomes much more simplified and one can easily deduce the consequences for different hierarchies of neutrino masses for the texture 6 zero as well as texture 5 zero mass matrices. Further, it may be added that the ranges of $D_{\nu}$ and $D_{l}$ taken here, suggest that the present mass matrices can be considered as 'natural' as advocated by Peccei and Wang [37]. This analysis also brings forth an important point that even though the neutrino mixing 
(a)

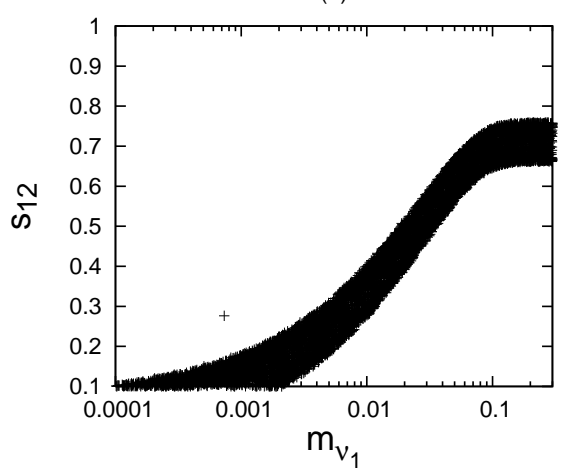

(c)

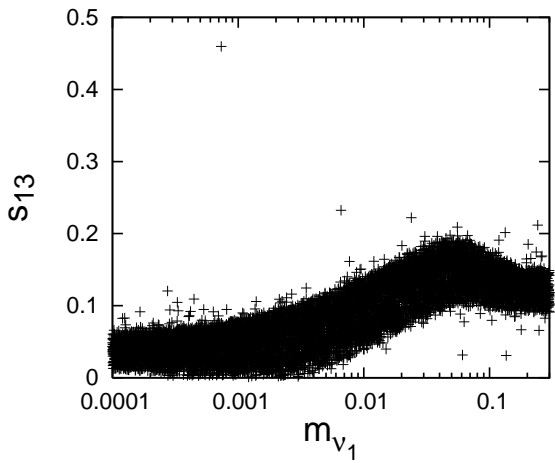

(b)

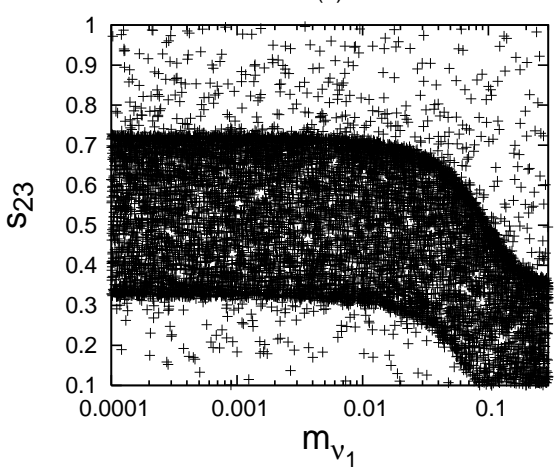

Figure 8: Plots showing variation of mixing angles $s_{12}, s_{13}$ and $s_{23}$ with the lightest neutrino mass $m_{\nu_{1}}$ for texture 5 zero lepton mass matrices when $D_{l} \neq 0$ and $D_{\nu}=0$.

pattern is very different from the quark mixing pattern, yet both can be described by 'natural' texture specific mass matrices.

To summarize, as a first step we have extended the very recent analysis of FSTY [26] regarding compatibility of texture 6 zero hermitian lepton mass matrices with the leptonic mixing data. Apart from reproducing their results we have been able to clearly rule out inverted hierarchy and degenerate scenario of neutrino masses in texture 6 zero mass matrices. Further, keeping in mind quark-lepton universality [29], in the present work we have carried out the analysis for the texture 5 zero lepton mass matrices also as these are not ruled out in the case of quarks, unlike the case of texture 6 zero Fritzsch-like and non Fritzsch-like quark mass matrices. Interestingly, again inverted hierarchy and degenerate scenario of neutrino masses for texture 5 zero mass matrices also seem to be ruled out.

The present analysis indicates that the present $3 \sigma$ C.L. range of $\theta_{13}$ does not put a reasonable constraint on the value of lightest neutrino mass $m_{\nu_{1}}$, therefore, refinements of 
its value will have important implications for $m_{\nu_{1}}$. Regarding, the effective Majorana mass $\left\langle m_{e e}\right\rangle$, one finds that for texture 5 zero lepton mass matrices when $D_{l}=0$ and $D_{\nu} \neq 0$ the $1 \sigma$ C.L. range of the mixing angle $\theta_{13}$ constrains the value of $\left\langle m_{e e}\right\rangle$ to be $2.3-8.7 \mathrm{meV}$. This range looks to be somewhat expanded in comparison to the one obtained in the case of texture 6 zero mass matrices due to the additional parameter $D_{\nu}$. Therefore, it seems that measurements of $m_{\nu_{1}}$ and $\left\langle m_{e e}\right\rangle$ would have important implications on texture specific mass matrices considered here.

\section{Acknowledgements}

P.F. would like to thank University Grants Commission (Ref. No: F. 4-3/2006(BSR)/589/2007(BSR)) for financial support. G.A. would like to acknowledge DST, Government of India (Grant No: SR/FTP/PS-017/2012) for financial support. P.F., S.S., G.A. acknowledge the Chairman, Department of Physics, P.U., for providing facilities to work. R.V. would like to thank the Director, RIEIT for providing adequate facilities.

\section{References}

[1] R. Davis, Prog. Part. Nucl. Phys. 32 (1994) 13.

[2] B. T. Cleveland, et al., HOMESTAKE Collaboration, Astrophys. J 496 (1998) 505.

[3] W. Hampel, et al., GALLEX Collaboration, Phys. Lett. B 57447 (1999) 127.

[4] M. Altmann, et al., GNO Collaboration, Phys. Lett. B 616 (2005) 174, hep-ex/0504037.

[5] J. P. Cravens, et al., SUPER-KAMIOKANDE Collaboration, Phys. Rev. D 78 (2008) 032002, arXiv:0803.4312.

[6] J. N. Abdurashitov, et al., SAGE Collaboration, Phys. Rev. C 80 (2009) 015807, arXiv:0901.2200.

[7] B. Aharmim, et al., SNO Collaboration, Phys. Rev. C 81 (2010) 055504, arXiv:0910.2984.

[8] R. Wendell, et al., SUPER-KAMIOKANDE Collaboration, Phys. Rev. D 81 (2010) 092004, arXiv:1002.3471.

[9] A. Gando, et al., KamLAND Collaboration, Phys. Rev. D 83 (2011) 052002, arXiv:1009.4771. 
[10] M. Appolonio, et al., CHOOZ Collaboration, Eur. Phys. J C 27 (2003) 331, hep-ex/0301017.

[11] L. Whitehead, MINOS Collaboration, Recent results from MINOS, Joint Experimental- Theoretical Seminar (24 June 2011, Fermilab, USA). Websites: theory.fnal.gov/jetp, http://www-numi.fnal.gov/pr plots/.

[12] M. H. Ahn, et al., K2K Collaboration, Phys. Rev. D 74 (2006) 072003, hep-ex/0606032.

[13] A. Habig, MINOS Collaboration, Mod. Phys. Lett. A 25 (2010) 1219, arXiv:1004.2647.

[14] P. Adamson, et al., MINOS Collaboration, Phys. Rev. Lett. 107 (2011) 181802, hep$\mathrm{ph} / 1108.0015$.

[15] K. Abe, et al., T2K Collaboration, Phys. Rev. Lett. 107 (2011) 041801, arXiv:1106.2822.

[16] Y. Abe, et al., DOUBLE-CHOOZ Collaboration, arXiv:1112.6353.

[17] F. P. An, et al., DAYA-BAY Collaboration, Phys. Rev. Lett. 108 (2012) 171803, arXiv:1203.1669.

[18] J. K. Ahn, et al., RENO Collaboration, arXiv:1204.0626.

[19] M. Appolonio, et al., CHOOZ Collaboration, Phys. Lett. B 466 (1999) 415, hep-ex/9907037.

[20] L. J. Hall, H. Murayama, and N. Weiner, Phys. Rev. Lett. 84 (2000) 2572, hep-ph/9911341.

[21] A. de Gouvea and H. Murayama, Phys. Lett. B 573 (2003) 94-100, hep-ph/0301050.

[22] G. Altarelli, et al., hep-ex/1207.0587.

[23] S. Weinberg, in Transactions of the New York Academy of Sciences 38 (1977) 185.

[24] H. Fritzsch, Phys. Lett. B 70 (1977) 436;

H. Fritzsch, Phys. Lett. B 73 (1978) 317. 
[25] H. Fritzsch, Z. Z. Xing, Prog. Part. Nucl. Phys. 45 (2000) 1, and references therein; Z. Z. Xing, Int. Jour. of Mod. Phys. A 19 (2004) 1, and references therein, hep-ph/9912358;

M. Gupta, G. Ahuja, Int. Jour. of Mod. Phys. A 26 (2011) 2973;

M. Gupta, G. Ahuja, Int. Jour. of Mod. Phys. A 27 (2012) 1230033 .

[26] M. Fukugita, et al., Phys. Lett. B (2012), hep-ph/1204.2389.

[27] M. Fukugita, et al., Phys. Lett. B 562 (2003) 273, hep-ph/0303177.

[28] N. Mahajan, R. Verma, M. Gupta, Int. Jour. of Mod. Phys. A 25 (2010) 1, hep$\mathrm{ph} / 0909.4843$.

[29] M. A. Schmidt, A. Yu. Smirnov, Phys. Rev. D 74 (2006) 113003 .

[30] R. Verma, G. Ahuja, N. Mahajan, M. Randhawa, M. Gupta, Journal of Physics G 37 (2010) 075020, arXiv:1004.5452;

R. Verma, G. Ahuja, M. Gupta, Phys. Lett. B 681 (2009) 330, arXiv:0909.4363;

G. Ahuja, M. Gupta, M. Randhawa, R. Verma, Phys. Rev. D 79 (2009) 093006, arXiv:0904.4534;

G. Ahuja, S. Kumar, M. Randhawa, M. Gupta, S. Dev, Phys. Rev. D 76 (2007) 013006, hep-ph/0703005;

M. Randhawa, G. Ahuja, M. Gupta, Phys. Rev. D 65 (2002) 093016, hep-ph/0203109.

[31] M. Fukugita, M. Tanimoto, and T. Yanagida, Phys. Lett. B 562 (2003) 273;

M. Fukugita, M. Tanimoto, and T. Yanagida, Prog. Theor. Phys. 89 (1993) 263.

[32] S. Zhou, Z. Z. Xing, Eur. Phys. J. C 38 (2005) 495;

Z. Z. Xing, invited talk at given at NOON (2004), hep-ph/0406049;

Z. Z. Xing, S. Zhou, Phys. Lett. B 593 (2004) 156;

Z. Z. Xing, S. Zhou, Phys. Lett. B 606 (2005) 145.

[33] G. L. Fogli, E. Lisi, A. Marrone, A. Palazzo and A. M. Rotunno, Phys. Rev. D 86 (2012) 013012, hep-ph/1205.5254.

[34] M. Randhawa, G. Ahuja, M. Gupta, Phys. Lett. B 643 (2006) 175, hep-ph/0607074.

[35] S. M. Bilenky, et al., hep-ph/1210.1306.

[36] C. Jarlskog, Phys. Rev. Lett 55 (1985) 1039;

C. Jarlskog, Phys. Rev. D 35 (1986)1685 ; 
C. Jarlskog, in CP violation, Ed. C. Jarlskog, World Scientific Publishing Co. Pte. Ltd, 1989 and references therein.

[37] R. D. Peccei and K. Wang, Phys. Lett. D 53 (1996) 2712, hep-ph/9509242. 